\title{
Stability of ageing-induced multiple-stage transformation behaviour in a Ti-50.15\% Ni alloy
}

\author{
Y. Liu ${ }^{1}$ and D. Favier ${ }^{2}$
}

\author{
Department of Mechanical and Materials Engineering, University of Westem Australia, \\ Nedlands, WA 6009, Australia \\ ${ }^{1}$ Laboratoire Sols, Solides, Structures, UMR 5521 du CNRS, Université Joseph Fourier, BP. 53, \\ 38041 Grenoble cedex, France
}

\begin{abstract}
A fully annealed Ti-50.15\% Ni alloy was found to develop a multiple-stage transformation behaviour after ageing at $623 \mathrm{~K}$. The transformation behaviour corresponded to the R-phase transition and two separate martensitic transformations on cooling and two reverse transformations of the two martensites on heating. This study investigated the effects of overheating and thermal cycling on the stability of the multiple-stage transformation behaviour. It was found that overheating to above $723 \mathrm{~K}$ for a short time restored the single-stage transformation behaviour by eliminating the second martensitic transformation whilst merging the R-phase transition into the first martensitic transformation. Thermal cycling, on the other hand, was found to cause a degradation of the multiplestage transformation behaviour by eliminating the first martensitic transformation.
\end{abstract}

\section{INTRODUCTION}

It has been reported in the literature that near equiatomic NiTi alloys may develop multiple-stage transformations during ageing treatment [1-7]. The transformation behaviour has been generally observed as three transformation stages on cooling and two transformation stages on heating by a variety of experimental techniques including differential thermal analysis [2], differential scanning calorimetry (DSC) [3,4], electrical resistance measurement [5], internal friction measurement [1], and shape memory measurement [3]. The three stages of the transformation on cooling are recognised as the Austenite-to-R phase transition and two separate martensitic transformations. On heating, the martensites transform back to austenite in two distinct stages, indicating that the two martensitic transformations are not successive transformations but two separate transformations. The temperature difference between the two reverse transformations is generally observed to be much smaller than that between the two forward martensitic transformations. These observations may be classified into two groups according to composition of the alloy and ageing conditions: $\mathrm{Ni}$-rich alloys $(>50.6 \mathrm{at} \% \mathrm{Ni})$ aged at above $750 \mathrm{~K}[2,5,6]$ and near-equiatomic $\mathrm{NiTi}(<50.2 \mathrm{at} \% \mathrm{Ni})$ aged at below $650 \mathrm{~K}[3,4,7]$.

Three main hypotheses have been proposed for this unusual transformation behaviour of NiTi alloys. Zhu and Gotthardt suggested that occurrence of anti-phase zones of vacancy ordering caused by ageing may be responsible for the separation of two martensitic transformations observed in a Ti49.86at\% Ni alloy after an ageing treatment at $773 \mathrm{~K}$ for $12.6 \mathrm{ks}$ [1]. This hypothesis is supported by the observation that ageing of a low-temperature-annealed sample, which was considered to contain a low vacancy concentration, did not induce the multiple-stage transformation behaviour in the alloy. Stroz et al. [8] suggested that some ageing-induced specific arrangements of dislocations hinder the martensitic transformation in certain areas, causing the separation of the two martensitic transformations. Favier et al. observed similar multiple-stage transformation behaviour in a Ti-49.5at\% $\mathrm{Ni}$ alloy after an ageing treatment at $623 \mathrm{~K} \mathrm{[3].} \mathrm{They} \mathrm{attributed} \mathrm{the} \mathrm{multiple-stage} \mathrm{transformation} \mathrm{behaviour} \mathrm{to} \mathrm{ageing-induced} \mathrm{precipitates.} \mathrm{It}$ is suggested that an ageing-induced precipitate imposes a stress-field within its vicinity. The matrix within the effective range of this stress field transforms to martensite at a different temperature from the rest, which is relatively unaffected. Bataillard et al. [9] provided transmission electron microscopic evidences demonstrating the effect of local stress fields around $\mathrm{Ni}_{4} \mathrm{Ti}_{4}$ precipitates on the multiple-stage martensitic transformation behaviour of a Ti-51.14at\% Ni alloy aged at $793 \mathrm{~K}$ for $1.8 \mathrm{ks}$. This investigation appears to have proven the precipitate hypothesis for the ageing-induced multiple-stage transformation behaviour observed in Ni-rich binary NiTi alloys.

McCormick et al. [4] and Liu et al. [7] observed the multiple-stage transformation in a Ti$50.2 \mathrm{at} \% \mathrm{Ni}$ alloy after ageing at $623 \mathrm{~K}$ for prolonged time, e.g., $>100 \mathrm{ks}$. These ageing conditions are distinctively different from those for $\mathrm{Ni}$-rich $\mathrm{NiTi}$ alloys. Although attributing the effect of ageing to the development of precipitates, the authors could not identify any ageing-induced precipitates with multiple attempts, even in over-aged specimens. Near-equiatomic NiTi alloys of $\mathrm{Ni}<50.2$ at $\%$ are not known in the literature to be able to develop precipitates during ageing [10]. The hypothesis of precipitates is only supported by the observation [7] of an "all-round shape memory effect", which is known to be associated 
with the development of ageing-induced precipitates [11]. In this regard, the mechanisms responsible for the development of multiple-stage transformation behaviour in these alloys are yet to be identified. Not aiming to clarify the mechanisms, this study was carried out to collect more experimental evidence for the characteristics of the multiple-stage transformation behaviour on its stability. The experiment was performed with respect to overheating and thermal cycling.

\section{EXPERIMENTAL DETAILS}

A Ti-50.15at\% Ni alloy in plate form was used in this study. The as-received material was first solution treated at $1213 \mathrm{~K}$ for $1.8 \mathrm{ks}$. The solution treated plate was cold rolled and then fully annealed for recrystalisation. Following the heat treatment, the samples were aged at $623 \mathrm{~K}$. The aged samples were polished to remove the oxidation surfaces and cut into $\sim 30 \mathrm{mg}$ cubes for DSC measurement. DSC measurement was carried out on a Perkin-Elmer DSC-7 in a He atmosphere with a heating/cooling rate of $10 \mathrm{~K} / \mathrm{min}$. The transformation behaviour of the samples was studied with respect to two post-ageing treatments: overheating and thermal cycling. Overheating was carried out in DSC to temperatures a few hundred degrees above the finish temperature of the reverse transformation. The cooling/heating rate for the overheating treatment was $100 \mathrm{~K} / \mathrm{min}$. Thermal cycling was performed between two liquid baths: boiling water and liquid-nitrogen-chilled methanol.

\section{RESULTS AND DISCUSSION}

\subsection{Ageing-induced multiple-stage transformation behaviour}

Fig. 1 shows the DSC measurement of the effect of ageing treatment on transformation behaviour for specimens annealed under different conditions. Curve (a) shows the transformation behaviour of a specimen annealed at $873 \mathrm{~K}$, which is representative of all specimens annealed in the temperature range between $873 \mathrm{~K}$ and $1273 \mathrm{~K}$ [12]. The specimen showed typical single-stage transformation behaviour both on cooling and on heating. The characteristic temperatures, as measured at the maximum heat flow, were determined to be $T_{M}=298 \mathrm{~K}$ for the austenite-to-martensite $(A \rightarrow M)$ transformation on cooling and $T_{A}=332$ $\mathrm{K}$ for the martensite-to-austenite $(\mathrm{M} \rightarrow \mathrm{A})$ transformation on heating. The transformation heat was measured to be $24.6 \mathrm{~J} / \mathrm{g}$ for $\mathrm{A} \rightarrow \mathrm{M}$ transformation and $26.5 \mathrm{~J} / \mathrm{g}$ for $\mathrm{M} \rightarrow \mathrm{A}$ transformation.

Specimens (b)-(e) were aged at $623 \mathrm{~K}$ for $259.2 \mathrm{ks}$ (72 hours). Specimen (b) had been annealed at $873 \mathrm{~K}$ and cooled to room temperature slowly in furnace prior to the ageing treatment. Specimen (c) had been annealed at the same temperature but quenched in water after the annealing. Specimens (d) and (e) were annealed at $1213 \mathrm{~K}$ and cooled in furnace and by quenching, respectively. It is seen that the four specimens showed almost identical transformation behaviour, indicating that prior annealing conditions had no influence on the effect of ageing. This observation does not appear to support the vacancy-ordering hypothesis.

These specimens exhibited three transformation stages on cooling and two transformation stages on heating. The peaks are identified as $\mathrm{A} \rightarrow \mathrm{R}$ transition and two martensitic transformations on cooling, as labelled $R, M 1$ and $M 2$, and two reverse transformations of the two martensites on heating, as labelled $\mathrm{A} 1$ and $\mathrm{A} 2$ [7]. The notation of $\mathrm{M} 1, \mathrm{M} 2, \mathrm{~A} 1$ and $\mathrm{A} 2$ is with no intention to suggest any difference

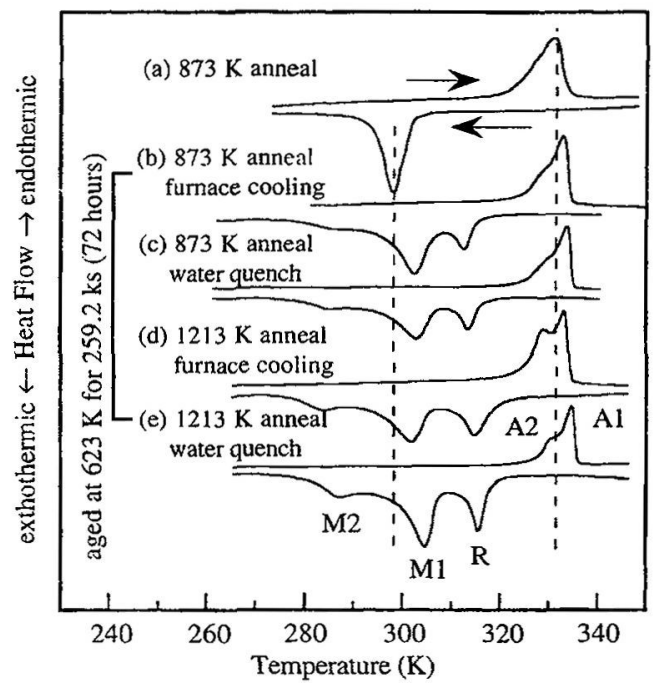

Fig.1 Effect of ageing on transformation behaviour in crystal structure between the two martensites or the two austenites. It is seen that after ageing the Rphase transition appeared and the martensitic transformation split into two transformations appearing on either side of its original position. On heating, the reverse transformation also split into two stages on either 
side of its original position, in a similar manner to the martensitic transformation except that the temperature difference between the two reverse transformations was much smaller than that between the two martensitic transformations. The characteristic temperatures for the transformations were determined to be $T_{R}=316 \mathrm{~K}$, $\mathrm{T}_{\mathrm{M} 1}=304 \mathrm{~K}, \mathrm{~T}_{\mathrm{M} 2}=287 \mathrm{~K}, \mathrm{~T}_{\mathrm{A} 2}=330 \mathrm{~K}$ and $\mathrm{T}_{\mathrm{Al}}=334 \mathrm{~K}$ (for curve (e)). The total transformation heats for the forward and the reverse transformations were both measured to be $22.3 \mathrm{~J} / \mathrm{g}$.

The ageing-induced multiple-stage transformations in near-equiatomic NiTi alloys have been identified to be $A \rightarrow R$ transformation (R), $A \rightarrow M$ transformation (M1) and $R \rightarrow M$ transformation (M2) in sequence on cooling and the $\mathrm{M} 2 \rightarrow \mathrm{A}(\mathrm{A} 2)$ and $\mathrm{M} 1 \rightarrow \mathrm{A}(\mathrm{A} 1)$ transformations in sequence on heating in a previous study [7]. The thermodynamic conditions of the multiple-stage transformations are schematically expressed in Fig.2, with (a) illustrating the as-annealed condition and (b) illustrating the as-aged condition. In this scheme the free energy status of martensite is represented using two lines, $\mathrm{M}$ - for cooling and $\mathrm{M}+$ for heating, to express the influence of the irreversible energy $\mathrm{E}_{\mathrm{j}}$, which gives rise to the transformation hysteresis $\eta$. (The thermal hysteresis of the R-phase transition is neglected in this scheme.) Under condition (a), austenite transforms to martensite on cooling at $T_{M}$ and martensite transforms back to austenite on heating at $\mathrm{T}_{\mathrm{A}}$.

In situation (b), the free energy of martensite (on cooling) is reduced relative to austenite, as indicated by $M-*$. The free energy of the $R$ phase is also reduced relative to austenite, as indicated by $R *$. The magnitude of the change for the $R$ phase is larger than that for martensite. As a result, austenite transforms to $R$ phase at $T_{R}$, austenite transforms to martensite at $T_{M 1}$ and $R$ phase transforms to martensite at $T_{M 2}$. In this case, $T_{R}>T_{M 1}>T_{M}>T_{M 2}$, which corresponds to the transformation sequence of the as-aged specimen. This suggests that the ageing treatment decreased free energy status of both martensite and the $R$ phase relative to austenite. The situation for the heating transformations is neglected both in the presentation of the figure and in the discussion for simplicity.
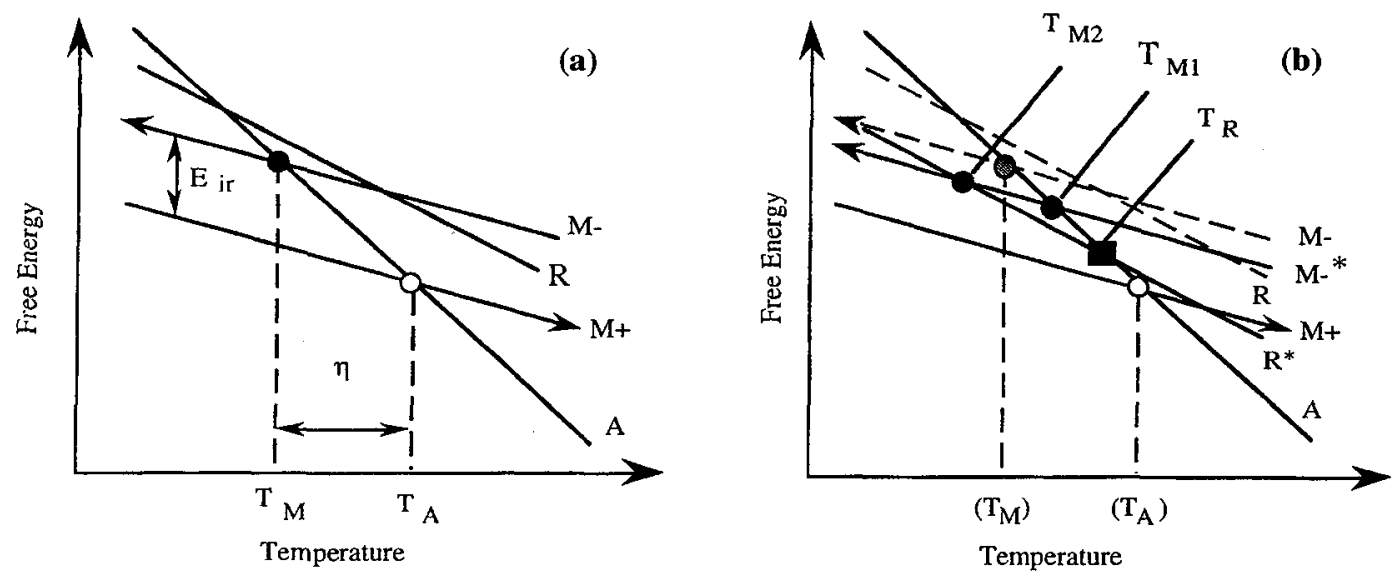

Fig.2 Schematic illustration of the effect of ageng on transformation sequence

\subsection{Effect of overheating}

The effect of overheating on the transformation behaviour of aged specimens was studied with respect to heating temperature and time. Fig.3 shows the effect of heating temperature on the transformation behaviour of a specimen annealed at $873 \mathrm{~K}$ prior to ageing. The overheating tests were performed rapidly with a heating/cooling rate of $100 \mathrm{~K} / \mathrm{min}$ and sequentially from low temperatures to high temperatures. There was no holding time at each heating temperature. It was observed that all transformations shifted to higher temperatures by a couple of degrees and then decreased slightly with increasing overheating temperature. It was also noticed that accompanying the small changes of transformation temperatures the relative intensity of $\mathrm{A} 2$ transformation increased initially and then decreased. However, it is evident that despite these moderate changes the multiple-stage transformation behaviour remained in style after overheating. Similar effects were also observed in other specimens. 
Fig. 4 shows the effect of holding time at $733 \mathrm{~K}$ for the transformation behaviour of an aged specimen. The transformation behaviour of an as-annealed specimen is also shown for comparison (curve (f)). It was observed that the relative intensity of the A2 transformation increased initially and then decreased to diminish with time. Accompanying the diminishing of the A2 transformation, M2 transformation also diminished after overheating for $1.02 \mathrm{ks}$. During the process, the characteristic temperatures of M1 and M2 remained unchanged. The R-phase transition, on the other hand, decreased in temperature to merge into M1. The surviving M1 transformation remained at the same temperature after overheating, $7 \mathrm{~K}$ above the original $\mathrm{T}_{\mathrm{M}}$, indicating that some permanent changes to the matrix had occurred after ageing. Despite the remarkable changes to the transformations, the total energies for both the heating reactions and the cooling reactions remained unchanged after the overheating, being $\sim 22 \mathrm{~J} / \mathrm{g}$.

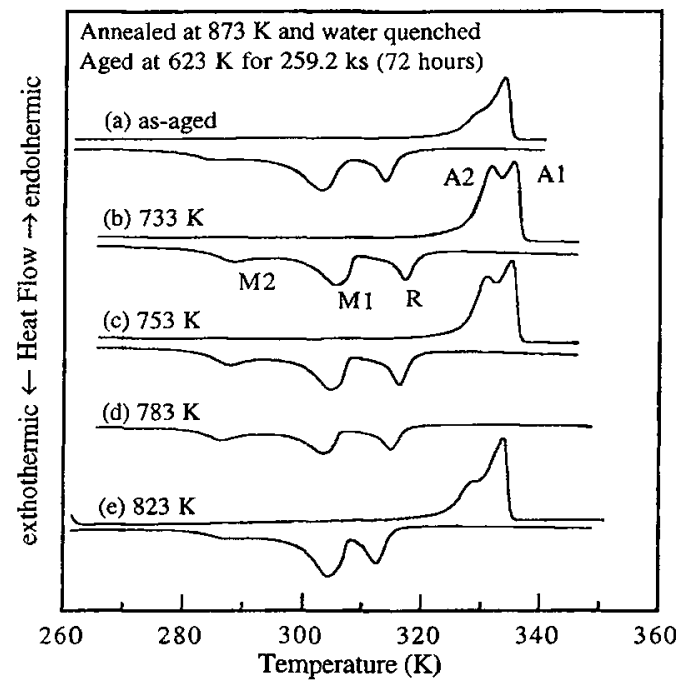

Fig.3 Effect of overheating temperature on transformation behaviour

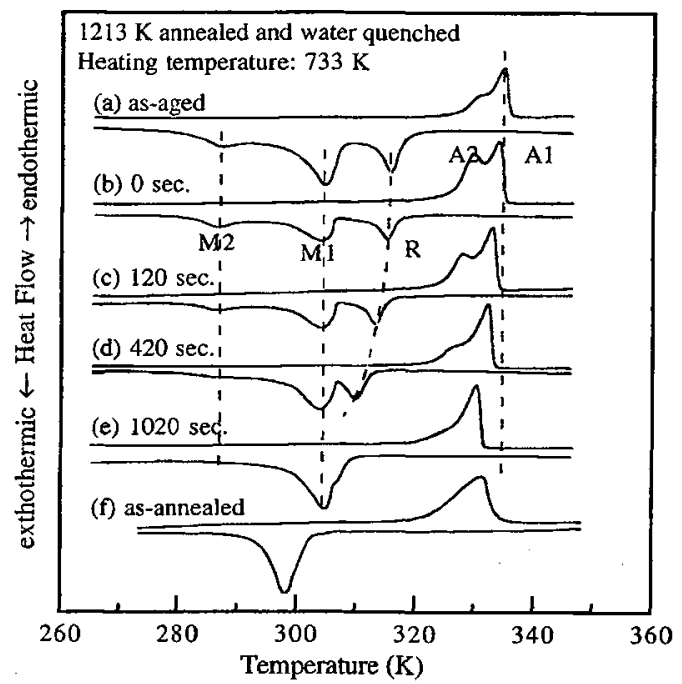

Fig.4 Effect of overheating time on transformation behaviour

Measurements of the maximum-heat-flow intensity ratio for A2 and A1 and for M2 and M1 are shown in Fig.5 with respect to time for three overheating temperatures. It is seen that there was an initial increase in $\mathrm{A} 2 / \mathrm{A} 1$ and $\mathrm{M} 2 / \mathrm{M} 1$ ratios with rapid heating to elevated temperatures. With prolonged heating, however, both $\mathrm{A} 2 / \mathrm{A} 1$ and $\mathrm{M} 2 / \mathrm{M} 1$ ratios decreased progressively with time, indicating the deterioration of the M2 and A2 transformations. The rate of the deterioration was faster at higher overheating temperatures. At $693 \mathrm{~K}$, the intensities of M2 and A2 transformations decreased only slightly relative to those of M1 and A1, respectively. This temperature may be identified as a threshold, below which the temperature has no significant influence on the multiple-stage transformation behaviour whereas above which degradation of M2 and A2 transformations occurs.

The results shown in Figs. 3 and 4 suggest that the deterioration of the multiple-stage transformation behaviour on overheating, more specifically the elimination of the R-phase, M2 and A2 transformations, was dependent on a diffusion process. If the R-phase transition and the associated M2 and A2 transformations are considered a direct result of ageing, overheating to above $733 \mathrm{~K}$ is seen to erase the effect of ageing. However, with regard to the fact that precipitates are formed in Ni-rich alloys at above $750 \mathrm{~K}$ [9], it is unlikely that the heating conditions, e.g., $733 \mathrm{~K}$ for $1.02 \mathrm{ks}$, were sufficient to dissolve any precipitates. This observation does not support the precipitate hypothesis.

\subsection{Effect of thermal cycling}

The effect of thermal cycling on the multiple-stage transformation behaviour is shown in Fig.6. The sample was annealed at $984 \mathrm{~K}$ prior to an ageing treatment at $625 \mathrm{~K}$ for $201.6 \mathrm{ks}$ ( 56 hours). It was observed that thermal cycling eliminated the M1 transformation while shifted it towards lower temperatures. As a consequence the exothermic heat intensity of the M2 transformation increased significantly. The R-phase transition remained largely unaffected during cycling. Corresponding to the disappearance of M1 on 
cooling, the intensity of transformation A1 on heating decreased to become negligible after cycling. The surviving transformation, A2, remained in the same temperature range. If $\mathrm{M} 2$ and $\mathrm{A} 2$ transformations are regarded as a direct consequence of the ageing treatment, thermal cycling appears to enhance the effect of ageing.

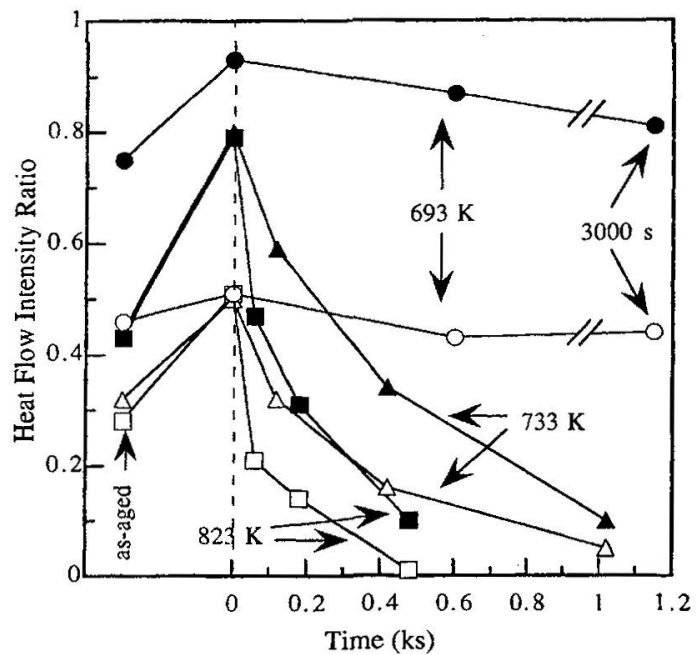

Fig.5 Evolution of transformation peak ratio during overheating

closed symbols: A2/A1; open symbols: M2/M1

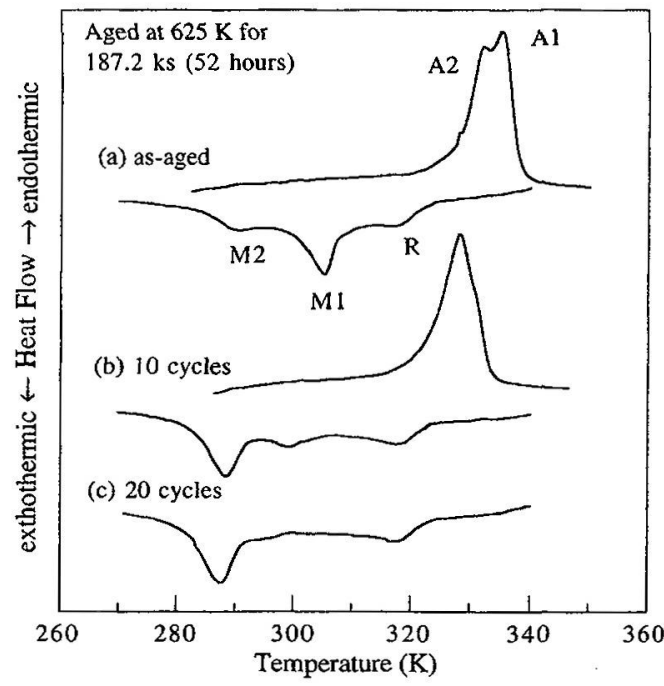

Fig.6 Effect of thermal cycling on transformation behaviour

The observations that overheating to $733 \mathrm{~K}$ erased the multiple-stage transformation behaviour and that thermal cycling enhanced it appear to defy the specific dislocation arrangement argument. If specific dislocation structures were formed during the ageing at $623 \mathrm{~K}$, it would be difficult to imagine that overheating to $100 \mathrm{~K}$ higher would destroy the specific structure of dislocations. Thermal cycling is expected to produce disordered dislocations.

\section{CONCLUSIONS}

The experimental evidences published in the literature appear to suggest that both the development of the multiple-stage transformation behaviour during ageing and the multiple-stage transformation behaviour developed are dependent on the composition for binary NiTi alloys. The mechanisms responsible for the ageing-induced multiple-stage transformation behaviour are thus expected not to be unique. The following conclusions are drawn for the $\mathrm{Ti}-50.15 \mathrm{at} \% \mathrm{Ni}$ alloy used in this study, which is believed to be representative for near-equiatomic $(\mathrm{Ni}<50.2 \mathrm{at} \%) \mathrm{NiTi}$ alloys:

(1) Conditions of pre-annealing exhibited no influence on the development of the multiple-stage transformation during ageing. This observation does not appear to support the vacancy-ordering hypothesis.

(2) The ageing-induced multiple-stage transformation behaviour was unstable at elevated temperatures above $733 \mathrm{~K}$. The deterioration of the behaviour was a time-dependent process and was faster at higher temperatures. The final transformation behaviour after overheating was a single $\mathrm{M} 1 \leftrightarrow \mathrm{A} 1$ transformation. These results are in direct contradiction to the observation of the development of precipitates at above $750 \mathrm{~K}$ for Ni-rich alloys. They do not appear to support the dislocationrearrangement hypothesis, either.

(3) Thermal cycling eliminates $\mathrm{Ml}$ and $\mathrm{A} 1$ transformations, resulting in a two-stage $\mathrm{A} \rightarrow \mathrm{R} \rightarrow \mathrm{M} 2$ transformation on cooling and a single reversion of $\mathrm{M} 2 \rightarrow \mathrm{A} 2$ on heating. This observation does not appear to support the dislocation-rearrangement hypothesis, based on the assumption that thermal transformation cycling introduces randomly distributed dislocations. However, this assumption is yet to be verified experimentally for specimens subjected to the ageing treatment. 


\section{References}

1 J.S. Zhu, R. Gotthardt, Phys. Lett. A 132, 279 (1988).

2. D. Stroz, J. Kwarciak, H. Morawiec, J. Mater. Sci. 23, 4127 (1988).

3. D. Favier, Yinong Liu, P.G. McCormick, Script Metall. Mater. 28, 669 (1993).

4. P.G. McCormick, Yinong Liu, X. Chen, D. Favier, Trans. Mat. Res. Soc. Jpn. 18B, 1105 (1994).

5. L. Bataillard, R. Gotthardt, in Proc. Int. Conf. Solid-Solid Phase Transformations, Pittsburgh, 1994, edited by W.C. Johnson, M. Howc, D.E. Laughlin, W.A. Soffa (TMS Warrendale, 1994), p.761.

6. L. Bataillard, R. Gotthardt, J. de Phys. IV 5, C8-647 (1995).

7. Yinong Liu, X. Chen, P.G. McCormick, J. Materials Sci. 32, 5979 (1997).

8. D. Stroz, Z. Bojarski, J. Ilczuk, Z. Lekston, H. Morawiec, J. Mater. Sci. 26, 1741 (1991).

9. L. Bataillard, J.-E. Bidaux, R. Gotthardt, Philosophical Magazine A 78, 327 (1998).

10. M. Nishida, C.M. Wayman, T. Honma, Metall. Trans. A 17A, 1505 (1986).

11. M. Nishida, C.M. Wayman, T. Honma, Scr. Metall. 18, 1389 (1984).

12. Yinong Liu, P.G. McCormick, ISIJ Int. 29, 417 (1989). 